\title{
DEL DISEÑO INSTRUCCIONAL AL DISEÑO DE APRENDIZAJE CON APLICACIÓN DE LAS TECNOLOGÍAS
}

Resumen: La tecnología incorporada a los procesos educativos puede ser un elemento enriquecedor y desarrollador para el aprendizaje; mas en aras de lograr esto, se hace necesario modificar los esquemas tradicionales de diseño y planificación de cursos y materiales para la enseñanza y utilizar las herramientas tecnológicas bajo una óptica abierta y de adaptación a los nuevos modelos de aprendizaje. El presente artículo tiene como objetivo realizar un recorrido por el diseño de aprendizaje, su historia y sus modelos. Para ello se aborda su origen y evolución y cómo las tendencias pedagógicas, sicológicas e históricas han modificado el curso del diseño de aprendizaje, educativo, o de aprendizaje que conocemos hoy. También se hace referencia a varios de los modelos que han existido, sus características fundamentales y las teorías de aprendizaje que los sustentan. Por último, se destacan algunas de las tendencias actuales del diseño de aprendizaje y su flexibilidad de acuerdo a los nuevos modelos y espacios de aprendizaje.

Palabras clave: diseño instruccional; diseño de aprendizaje; tecnologías.

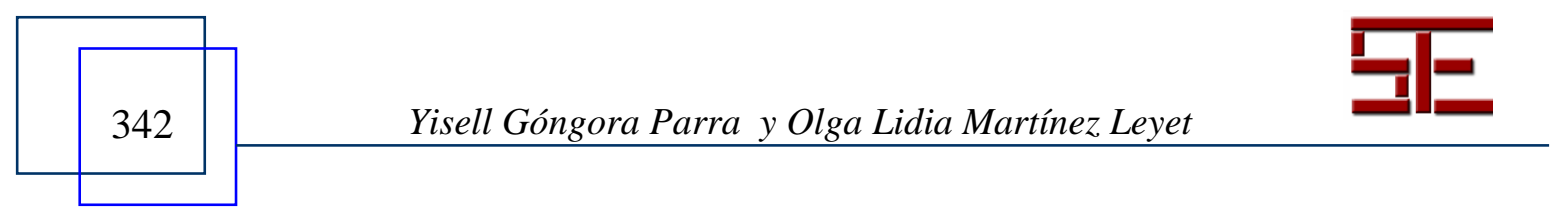




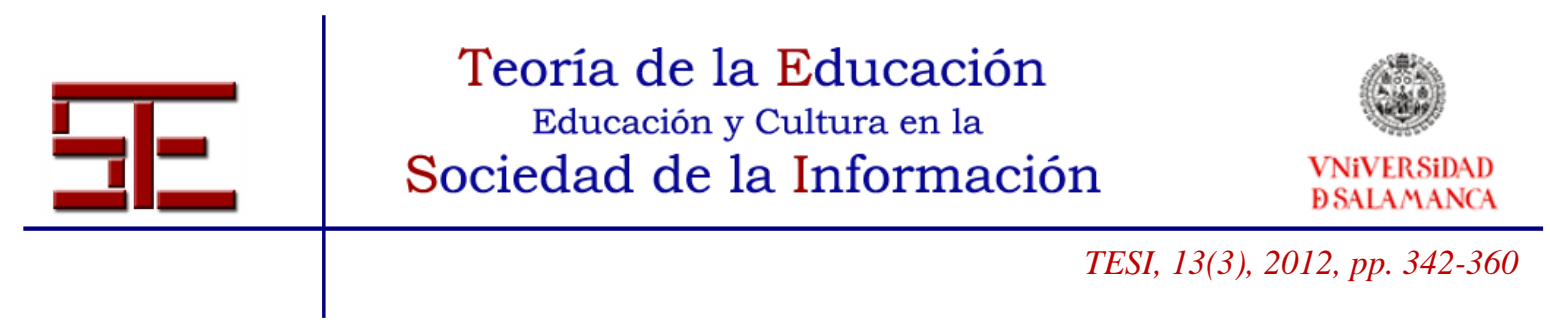

\title{
FROM INSTRUCTIONAL DESIGN TO TECNOLOGY BASED LEARNING DESIGN
}

\begin{abstract}
Technology incorporated to education has proved to foster and improve the teaching learning process; however, in order to achieve the best results it is necessary to change traditional paradigms for the design and planning of courses and teaching materials and use technological tools with open minds and flexibility to adjust to new learning environments. This paper aims at discussing learning design, its evolution and models, referring to its origins and development as well as to pedagogical, psychological and historical tendencies that have shaped its concept. The main models are also discussed, their characteristics and the learning theories that support them. Finally, this paper deals with current tendencies in learning design and its flexibility in new models and learning environments.
\end{abstract}

Keywords: instructional design; learning design; technologies.

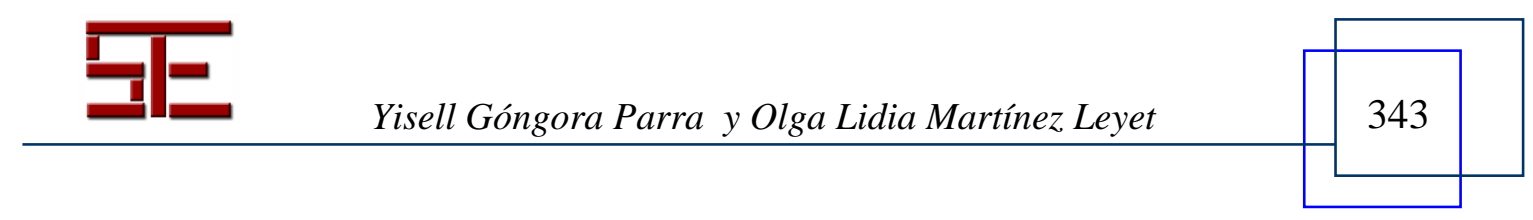




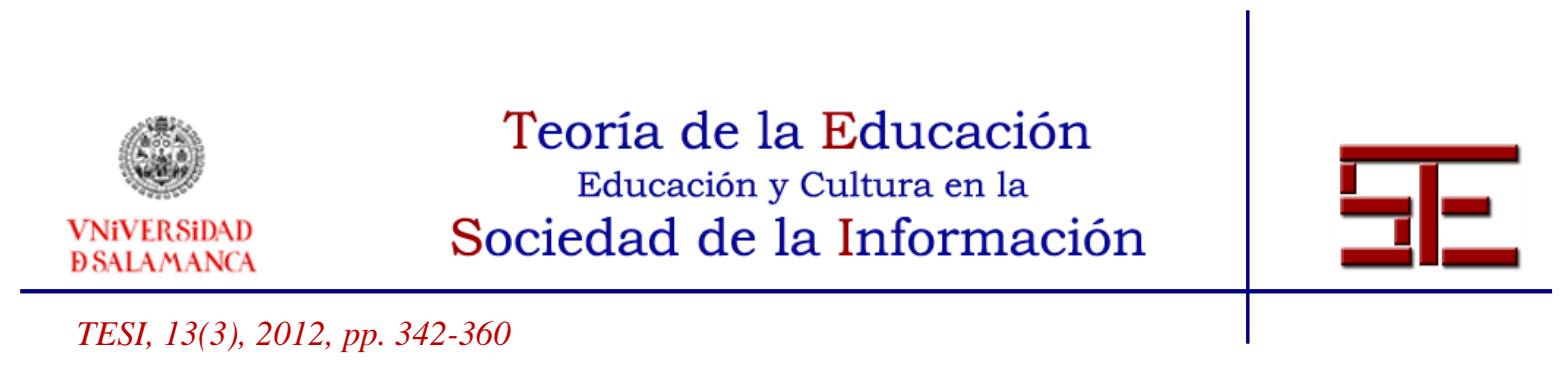

\section{DEL DISEÑO INSTRUCCIONAL AL DISEÑO DE APRENDIZAJE CON APLICACIÓN DE LAS TECNOLOGÍAS}

Fecha de recepción: 18/02/2012; fecha de aceptación: 28/10/2012; fecha de publicación: 30/11/2012

Yisell Góngora Parra

yisell@uci.cu

Universidad de las Ciencias Informáticas. Cuba.

Olga Lidia Martínez Leyet

olmartinez@uci.cu

Universidad de las Ciencias Informáticas. Cuba.

\section{INTRODUCCIÓN}

Ante el auge de las Tecnologías de la Información y las Comunicaciones se hace necesario modificar los esquemas tradicionales de diseño y planificación de cursos y materiales para la enseñanza y utilizar las herramientas tecnológicas bajo una óptica reflexiva y de adaptación a los nuevos modelos de aprendizaje. La mayoría de los docentes de experiencia hoy tuvieron su primera formación bajo los preceptos de la enseñanza tradicional y son, como define Marc Prensky (2008), "inmigrantes digitales" y no "nativos digitales"; por ello se tiene el gran reto, en la sociedad actual, de tener una actitud abierta y flexible ante el universo que abre la incorporación cada vez más activa de las tecnologías a la educación.

Los materiales y programas que se diseñen para el aprendizaje deben promover el desarrollo del pensamiento lógico y habilidades de pensamiento más efectivas. En el caso de la evaluación, exige la elaboración y uso de evaluaciones no tradicionales. Sin embargo, hay una condición sine qua non para lograr materiales y programas de calidad: la continua retroalimentación del impacto de estos en los estudiantes. Cada estudiante tiene su propio estilo de aprendizaje, por lo que resulta riesgoso guiarse solo por normas generales del diseño de materiales.

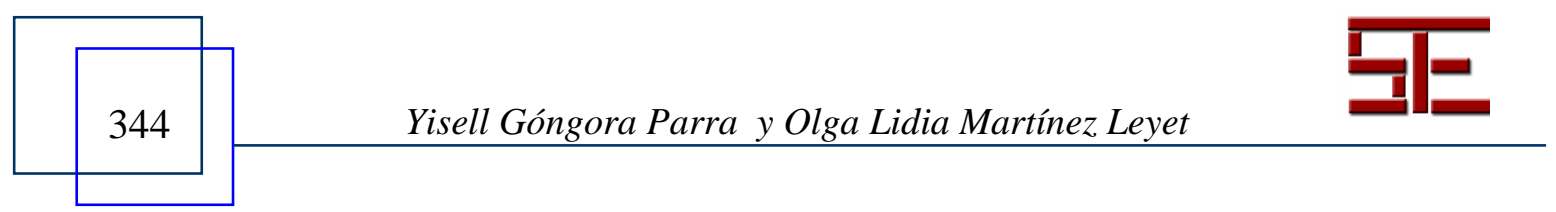




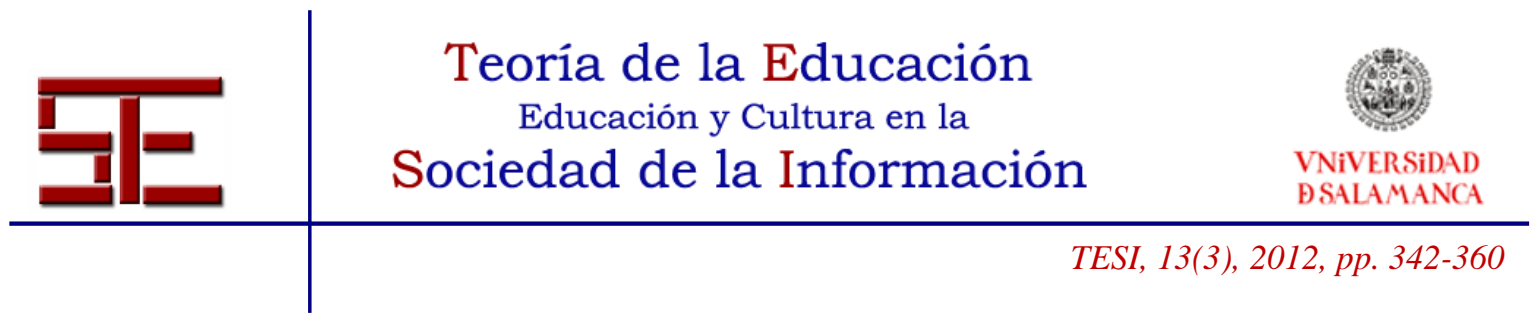

El diseño de aprendizaje se conoce también con los términos diseño instruccional o educativo (Koper, 2005). En la actualidad existen varios modelos de diseño instruccional usados para el diseño de cursos, programas y todo tipo de materiales para el aprendizaje, los cuales se abordan en este artículo. Unos siguiendo los preceptos de las teorías conductistas y cognitivistas y otros con un enfoque constructivista. Si apostamos por este último se requiere que el profesor o diseñador de aprendizaje produzca programas y materiales de naturaleza mucho más facilitadora que prescriptiva (Guàrdia, 2005).

Las nuevas tendencias del diseño de aprendizaje favorecen la no limitación de los contenidos de los cursos, la autonomía del que aprende para determinar la dirección del proceso y la evaluación como una categoría más subjetiva que no se rija por criterios cuantitativos específicos, sino que se evalúen los procesos y se dé protagonismo a la autoevaluación.

El diseño de instrucción como proceso tiene en cuenta los puntos débiles y fuertes de las diferentes teorías de aprendizaje, de esta forma puede tomar lo que necesite para lograr una planificación de calidad, que tribute al aprendizaje. El diseño de aprendizaje basado en estas teorías permite tener una visión holística del proceso de enseñanza aprendizaje, de esta forma es posible identificar las necesidades de los estudiantes y las nuevas oportunidades para un mejor aprovechamiento y motivación.

\section{DESARROLLO}

\section{1.- Origen y evolución del diseño de aprendizaje.}

Unos de los precursores del diseño de aprendizaje como lo conocemos hoy fue Edward Thorndike, hacia 1900. Fue uno de los iniciadores del conductismo y profesor de sicología educativa en la Universidad de Harvard. En las instituciones educacionales, venía ocurriendo un cambio de un conocimiento empírico hacia una base de conocimiento más educativa, basándose en los estudios realizados por Thorndike. Para él, el aprendizaje se basa en una serie de conexiones entre un estímulo y una respuesta. Estas teorías sobre la instrucción y el aprendizaje tienen una interpretación mecanicista y conductista.

Ya en la década de 1920 se comienza a considerar la relación entre las necesidades de la sociedad y la educación. Se desarrollaron planes de instrucción personalizada que

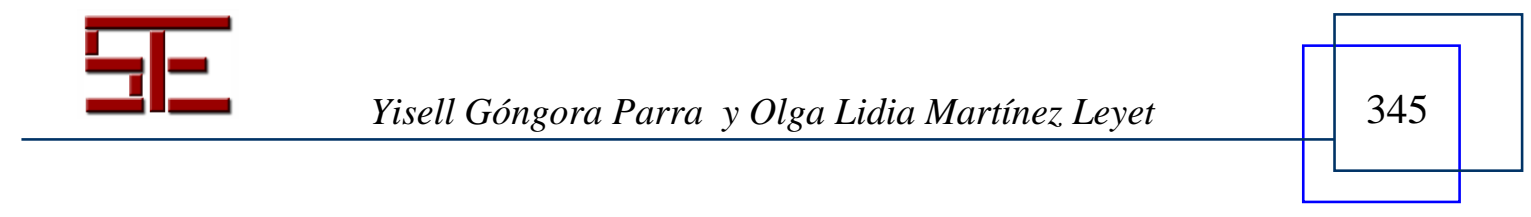




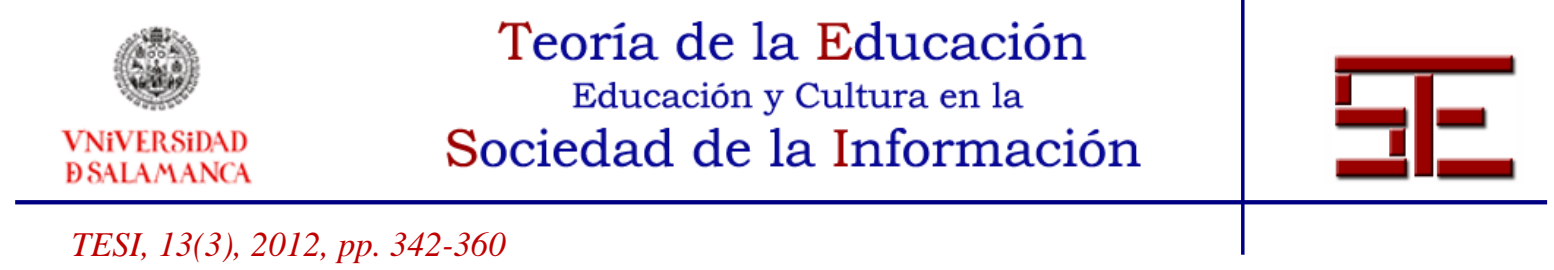

permitían a los aprendices ir a su propio ritmo con menos intervención por parte del profesor. Surge el contrato de aprendizaje y el aprendizaje experto, y las raíces de un análisis de trabajo y análisis de tareas (González, 2008). Constituye un gran paso de avance que la educación identifique más claramente su vinculación a la sociedad.

Hacia 1930, el plan de estudio de Tyler se convirtió en la base para especificar los objetivos generales y los objetivos conductuales. "Siempre que haya educación, habrá un plan de estudios" (Tyler, 1990).

Tyler se convirtió en precursor de la evaluación formativa, por sus ideas novedosas en este tema se considera el padre de la evaluación educativa. Hasta ese momento los conceptos evaluación y medición se usaban indistintamente, sin embargo, Tyler revoluciona estas teorías con sus ideas de que educar es más que transmitir conocimientos, es también cambiar los patrones de conducta de los estudiantes. De ahí su modelo de los objetivos de aprendizaje que tuvo un gran impacto en las concepciones del diseño curricular.

El diseño instruccional, entonces, aunque ya contemplaba una arista educativa y formativa, aún consideraba los exámenes como la única forma de evaluación eficaz para verificar el cumplimiento de los objetivos.

Durante la década del 40 se creó un equipo para desarrollo instruccional. Debido a la Segunda Guerra Mundial se hacían necesarias la formación y la capacitación acelerada de personal, sobre todo del ejército. Se utilizaron entonces estrategias tales como el uso de películas para entrenar. La década del 50 marca el surgimiento de la instrucción programada. El personal de la fuerza aérea fue el que inicialmente puso en práctica la teoría del aprendizaje avanzado y el análisis de tareas (Ruiz, 2007).

Los años 60 permearon al diseño instruccional de las teorías de la psicología cognitiva, sobre todo las de Gagné y Glase y el enfoque de sistemas de Finn. Hubo un auge en el diseño de materiales instruccionales. La década del 70 marca un desarrollo de las evaluaciones para comprobar el nivel de aprendizaje alcanzado por parte del estudiante. El diseño instruccional se sigue rigiendo por los principios del enfoque cognitivo y se destacan los trabajos de David Ausubel, Jerome Bruner y Robert Gagné, entre otras estrategias instruccionales (Ryder, 2006).

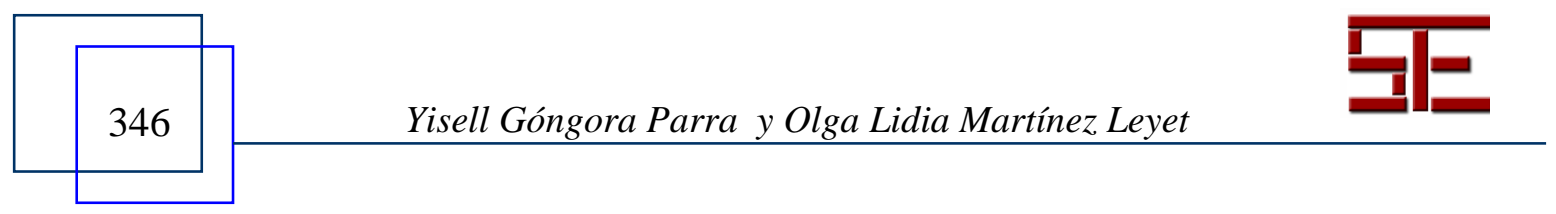




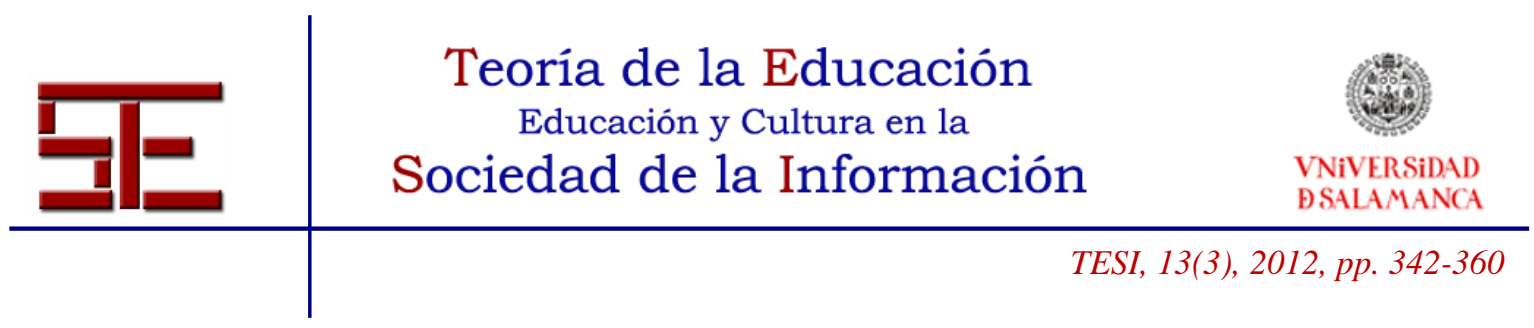

A Ausubel, por ejemplo, se le atribuye la teoría de aprendizaje de organizadores avanzados, que se aplicaba al aprendizaje de una segunda lengua, entre otras áreas. También se destacan en la época Leslie Briggs, Albert Bandura, Walter Dick y Benjamin Bloom, doctor en Educación de la Universidad de Chicago (USA), con su Taxonomía de Dominios del Aprendizaje, desde entonces conocida como Taxonomía de Bloom, que plantea que después de realizar un proceso de aprendizaje, el estudiante debe haber adquirido nuevas habilidades y conocimientos (Eduteka, 2003).

Ya en los 80 se trata de lograr un mayor acercamiento entre el desarrollo de la tecnología y el aprendizaje. Se realizan diseños en computadora que favorecieran la interactividad. Autores como Tyler (1986) enfatizan la diferencia que existe entre la relación de los elementos del diseño percibida por un experto en la materia y por un estudiante. Tyler señala que en el diseño instruccional se debe tratar de lograr que la organización lógica y el significado preciso que tienen los programas y cursos para un experto en la materia resulten también en una organización psicológica apropiada para el estudiante y su desarrollo intelectual.

Desde los años 90 hasta la actualidad proliferan los modelos de enfoque contructivista para el aprendizaje y los desarrollos multimedia. El hipertexto y los hipermedios, característicos del gran universo de información que representa Internet, señalan a estos modelos como los más apropiados para el diseño de aprendizaje. Siguiendo los preceptos de la teoría constructivista, se pueden introducir mejoras constantes en el proceso de aprendizaje. Además, adquiere gran importancia el concepto de evaluación formativa o continua y el de autoevaluación.

\section{2.- Modelos más representativos de diseño de aprendizaje}

Entre los primeros modelos de diseño instruccional estuvo el propuesto por Dick y Carey, originalmente en 1978, cuyos principios fundamentales eran básicamente conductistas. Este modelo describía todas las fases de un proceso iterativo que comenzaba por identificar los objetivos instruccionales y culminaba con la evaluación sumativa (Dick \& Carry, 2004).

Según este modelo lo primero consiste en identificar los objetivos en términos de comportamiento aprendido por el estudiante. Este comportamiento deseado se divide en habilidades subordinadas y se trabaja en la adquisición de estas. El aprendizaje del estudiante se constata mediante las habilidades que demuestre al final del proceso, haciendo énfasis en la capacidad de memorizar las respuestas y conceptos correctos.

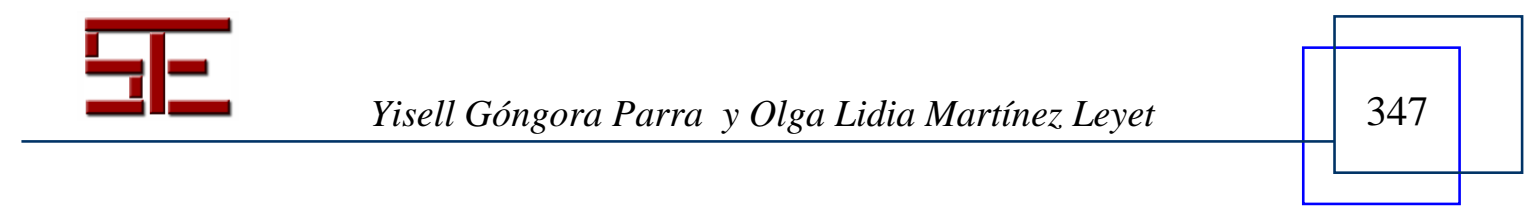




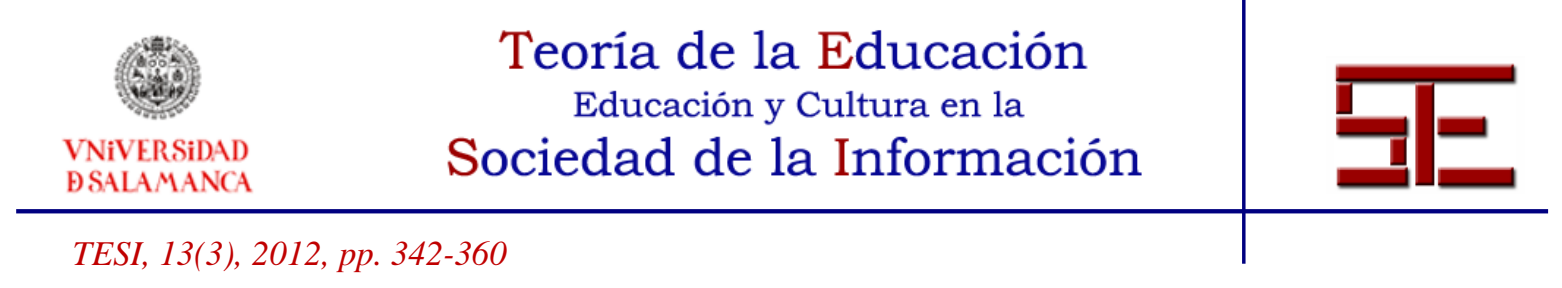

En 1980, el Dr. Vernon Gerlach, quien trabajaba en la Universidad del estado de Arizona, y el profesor Donald Ely, de la Facultad de Siracusa, proponen otro modelo. En este, la estructura del aprendizaje estaba basada en pasos; los objetivos debían ser específicos y la evaluación debía estar basada en el tema específico a tratarse (Gerlach y Ely, 1980).

Según este modelo los objetivos debían incluir las condiciones bajo las cuales el comportamiento ocurriría, así como los criterios para clasificarlo como aceptable. Para determinar la estrategia el profesor decidía cómo usar la información, determinaba los materiales y definía el rol del estudiante. El éxito del proceso de enseñanza-aprendizaje se medía por los cambios observables y medibles ocurridos en los estudiantes. Este modelo agrupa principios del conductismo y del cognitivismo.

En 1983 se crea el modelo de Merrill, conocido como Component Display Theory. Es un modelo que aún mantiene algunos de los preceptos conductistas de estímulorespuesta, pero incorpora también aspectos de la teoría cognitivista.

Este modelo clasifica el aprendizaje en dos dimensiones: el contenido (hechos, conceptos, procedimientos y principios) y el comportamiento (recordar, usar). Además, plantea que la instrucción es más efectiva cuanto más cerca observa las reglas, es decir, primeramente es necesario plantear los objetivos con claridad, seguidos de una combinación de reglas como ejemplificar, recordar, practicar y sugiere el uso de recursos mnemotécnicos apropiados para la tarea de aprendizaje (Merril, 1983).

Luego surge el modelo ASSURE, que es un modelo de Diseño Instruccional que los profesores pueden usar para diseñar y planear las clases. Sus siglas corresponden a Análisis de los estudiantes, Establecimiento (setting en inglés) de objetivos, Selección de métodos instruccionales, medios y materiales, Utilización de medios y materiales, Requerimiento de la participación del estudiante y Evaluación y revisión.

El modelo ASSURE incorpora los eventos de instrucción de Robert Gagné para asegurar el uso efectivo de los medios en la instrucción (Heinich, 1999). Es un modelo

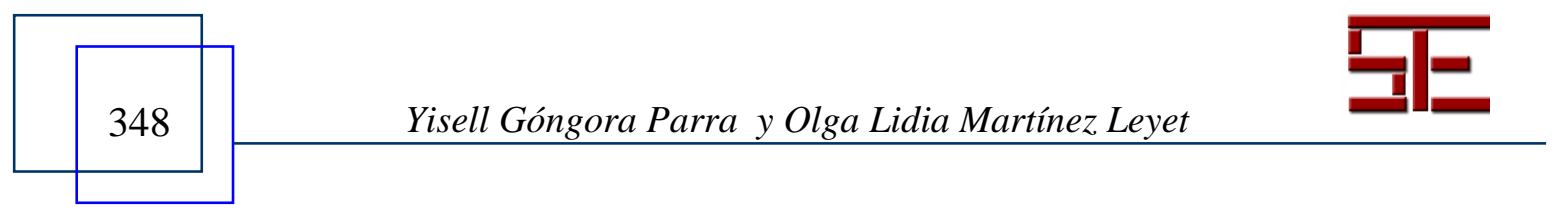




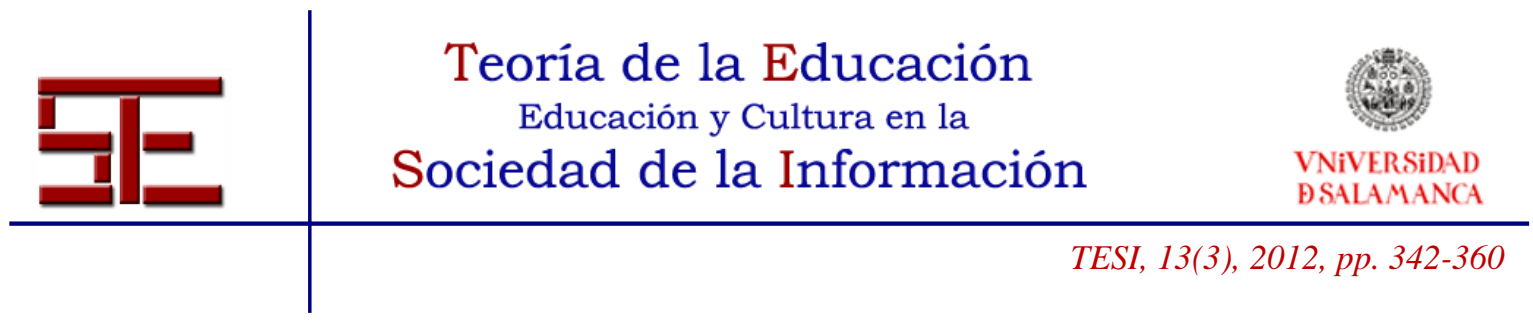

cognitivista que da más participación al estudiante para crear su experiencia de aprendizaje.

En 1985 se propuso el modelo de diseño de Jerold Kemp con su obra The Instructional Design Process. La forma oval de este modelo da al diseñador la sensación de que es un ciclo continuo que requiere de planificación, diseño, desarrollo y evaluación constantes para asegurar el proceso de aprendizaje efectivo.

El modelo es sistémico y no lineal y la teoría que lo sustenta es cognitivista, aunque luego ha desarrollado enfoques constructivistas. Se centra en las necesidades del estudiante, sus metas, prioridades y limitaciones y tiene en cuenta la evaluación formativa y sumativa (Kemp, 1985). El modelo está concebido para que cada fase tenga una revisión y es flexible para realizar adaptaciones o modificaciones, según sea conveniente.

En sus primeras etapas permite identificar los problemas instruccionales, especificar los objetivos, las necesidades de aprendizaje y trabajar para satisfacerlas. La motivación del estudiante es fundamental y tiene en cuenta los estilos de aprendizaje. Luego se realiza la selección de contenidos y se diseñan las tareas, estrategias didácticas y materiales. Finalmente se diseñan los instrumentos de evaluación.

A finales de los 80 se difundió el modelo de prototipado rápido, que repetía las etapas en forma de espiral. Este modelo exige un proceso de retroalimentación en el diseño de aprendizaje que se desarrolla en las siguientes fases: trabajo conceptual, análisis de necesidades, desarrollo, implementación de un primer sistema reducido (por ejemplo, un primer módulo del entorno de aprendizaje), evaluación, mejoramiento, evaluación, (Tripp et al., 1990). En el prototipado rápido se comienza desarrollando una pequeña área funcional de un entorno de aprendizaje, a fin de poder verificar el efecto y resultado final del proceso de desarrollo (Ballstaedt, 2000). Para ello es importante que el área seleccionada sea lo más representativa posible del total diseñado.

Este modelo ha sido exitosamente usado en la ingeniería de software en la actualidad.

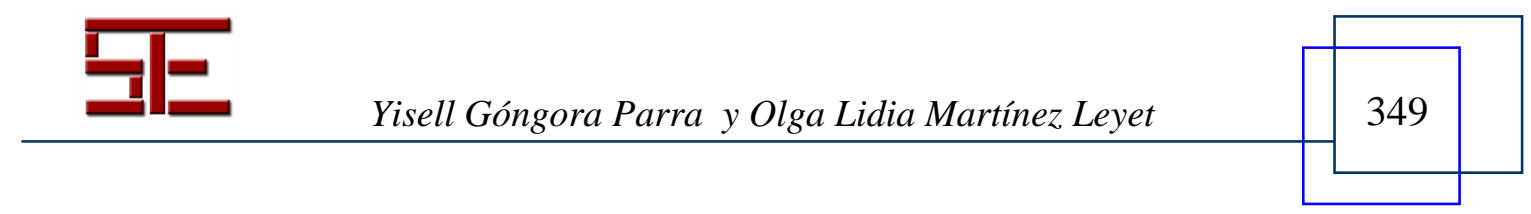




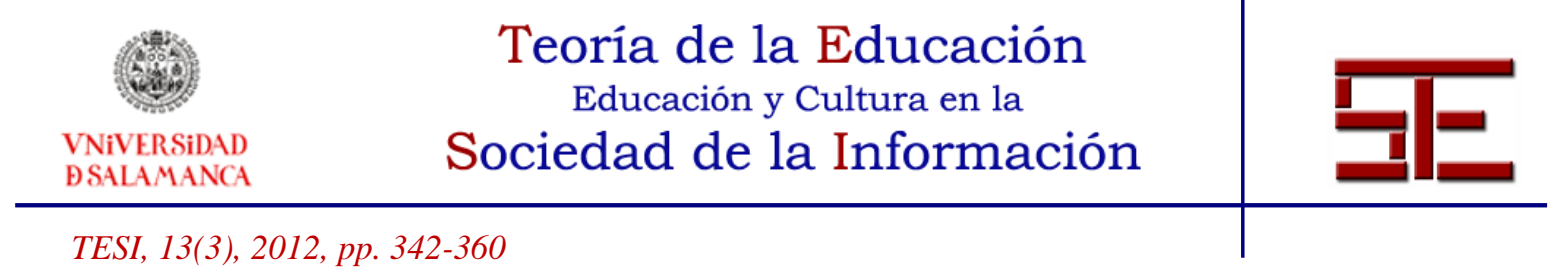

En 1999 el profesor Richard E. Mayer, del Departamento de Psicología de la Universidad de California, propuso el modelo SOI. Las siglas responden a Selección de la información relevante, Organización de la información de forma significativa para el estudiante y, por último, Integración de la nueva información con el conocimiento anterior del estudiante.

Este modelo plantea un enfoque constructivista para el diseño de textos que permite al estudiante construir resultados de aprendizaje significativos.

En esta misma época surge el modelo para el diseño de ambientes de aprendizaje constructivistas de Jonassen. Este enfatiza el papel del aprendiz en la construcción del conocimiento y aboga por que los estudiantes deben aprender haciendo. Propone el aprendizaje basado en la solución de problemas, casos o proyectos.

Primeramente el estudiante debe ubicarse en el contexto del problema, para luego hacerse una representación del mismo. Para que el estudiante esté motivado el problema debe ser interesante y atrayente. Luego debe crearse lo que Jonassen define como la manipulación del problema, donde los estudiantes tienen que construir un producto, manipular parámetros, tomar decisiones y afectar el ambiente de algún modo (Jonassen, 1999).

Este modelo promueve la importancia para el aprendizaje de que los estudiantes tengan acceso a casos relacionados, recursos de información y herramientas de colaboración.

Luego surge el modelo genérico ADDIE, compuesto por 5 etapas fundamentales cuyas iniciales forman su nombre: Análisis, Diseño, Desarrollo, Implementación y Evaluación. Los resultados de la evaluación formativa de cada fase pueden conducir al diseñador de aprendizaje de regreso a cualquiera de las fases previas. (Ver Tabla 1)

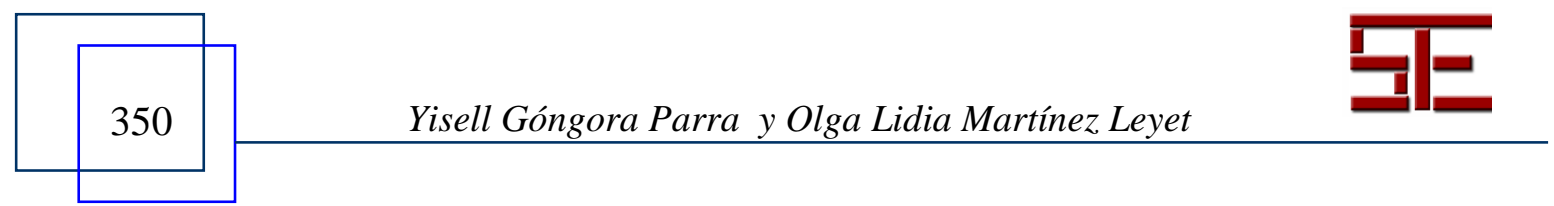




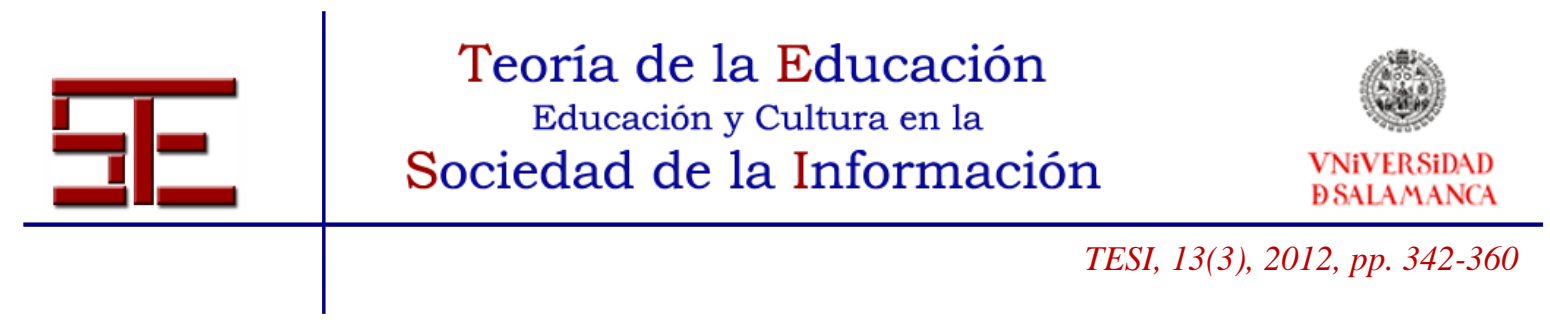

Tabla 1. Proceso de diseño de aprendizaje

\begin{tabular}{|c|c|c|}
\hline & Tareas & Resultados \\
\hline $\begin{array}{l}\text { Análisis } \\
\text { El proceso de definir qué } \\
\text { es aprendido }\end{array}$ & $\begin{array}{ll}\text { Evaluación } & \text { de } \\
\text { necesidades } & \\
\text { Identificación } & \text { del } \\
\text { Problema } & \\
\text { Análisis de tareas } & \\
\end{array}$ & $\begin{array}{l}\text { Perfil del estudiante } \\
\text { Descripción de obstáculos } \\
\text { Necesidades, definición de } \\
\text { problemas }\end{array}$ \\
\hline $\begin{array}{l}\text { Diseño } \\
\text { El proceso de especificar } \\
\text { cómo debe ser aprendido }\end{array}$ & $\begin{array}{l}\text { Escribir los objetivos } \\
\text { Desarrollar los temas a } \\
\text { evaluar } \\
\text { Planear la instrucción } \\
\text { Identificar los recursos } \\
\end{array}$ & $\begin{array}{l}\text { Objetivos medibles } \\
\text { Estrategia Instruccional } \\
\text { Especificaciones del } \\
\text { prototipo }\end{array}$ \\
\hline 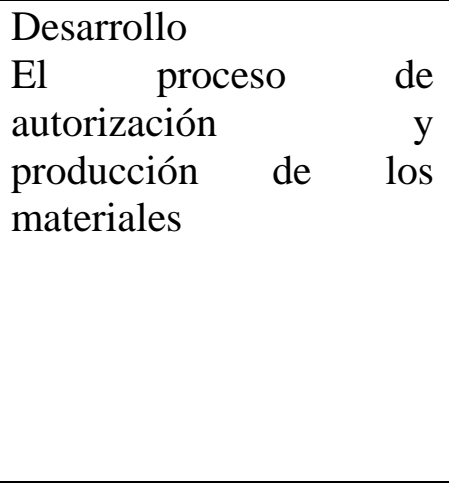 & $\begin{array}{l}\text { Trabajar con } \\
\text { productores } \\
\text { Desarrollar el libro de } \\
\text { trabajo, organigrama y } \\
\text { programa } \\
\text { Desarrollar los ejercicios } \\
\text { prácticos } \\
\text { Crear el ambiente de } \\
\text { aprendizaje }\end{array}$ & $\begin{array}{l}\text { Instrucción basada en la } \\
\text { computadora } \\
\text { Instrumentos } \\
\text { retroalimentación } \\
\text { Instrumentos de medición } \\
\text { Instrucción mediada por } \\
\text { computadora } \\
\text { Aprendizaje colaborativo } \\
\text { Entrenamiento basado en el } \\
\text { Web }\end{array}$ \\
\hline $\begin{array}{l}\text { Implementación } \\
\text { El proceso de instalar el } \\
\text { proyecto en el contexto } \\
\text { del mundo real }\end{array}$ & $\begin{array}{l}\text { Entrenamiento docente } \\
\text { Entrenamiento piloto }\end{array}$ & $\begin{array}{l}\text { ntarios del estudiante } \\
\text { de la evaluación }\end{array}$ \\
\hline $\begin{array}{l}\text { Evaluación } \\
\text { El proceso de determinar } \\
\text { la adecuación de la } \\
\text { instrucción }\end{array}$ & $\begin{array}{l}\text { Datos de registro del } \\
\text { tiempo } \\
\text { Interpretación de los } \\
\text { resultados de la } \\
\text { evaluación } \\
\text { Encuestas a graduados } \\
\text { Revisión de actividades }\end{array}$ & $\begin{array}{l}\text { Recomendaciones } \\
\text { Informe de la evaluación } \\
\text { Revisión de los materiales } \\
\text { Revisión del prototipo }\end{array}$ \\
\hline
\end{tabular}

(McGriff, 2000)

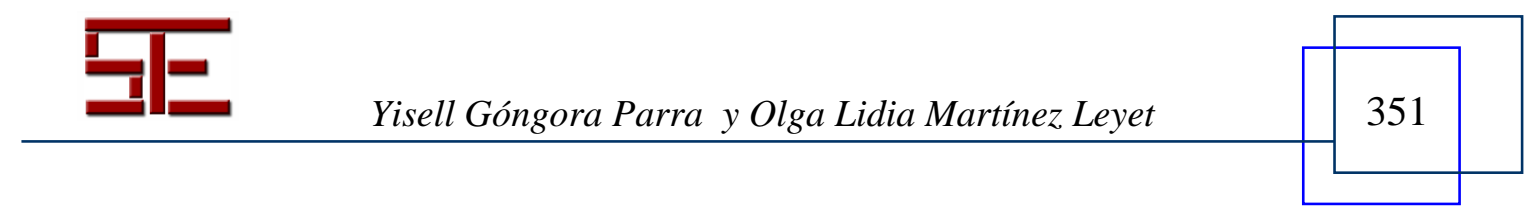




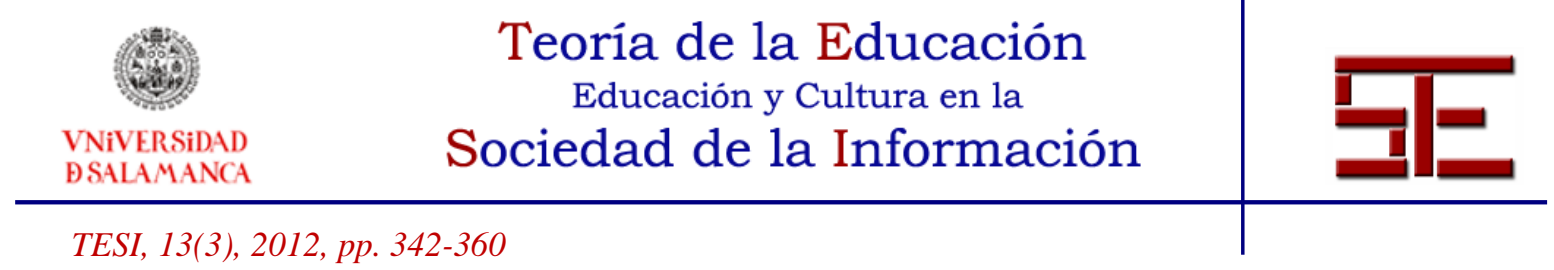

En el año 2003, luego de una revisión crítica al modelo ADDIE por Pedro S. Cookson, surge el modelo PRADDIE. El modelo ADDIE había sido criticado en ocasiones por ser cerrado y poco flexible, además de pasar por alto las consideraciones filosóficas, financieras, institucionales, entre otras.

Cookson añade al modelo ADDIE una fase de pre-análisis para evitar las limitaciones de un modelo cerrado, y esta apertura la señala con flechas de dos puntas interrelacionando las diferentes etapas del modelo; lo cual indica que estas relaciones se caracterizan por reciprocidad mutua. Aunque el diseño de aprendizaje puede desarrollarse en un orden racional y cronológico particular, también es posible que ocurran variaciones en la secuencia. Otra característica de este modelo es que la evaluación puede aplicarse a todas las demás fases, lo que garantiza la oportunidad de revisión, reflexión y cambio en cualquier etapa del proceso.

Las nuevas tendencias en el diseño de aprendizaje plantean que el profesor o diseñador debe producir programas y materiales de naturaleza mucho más facilitadora que prescriptiva (Guàrdia, L. 2005). Mientras que en los modelos lineales de diseño de enseñanza-aprendizaje solo existe un camino de planeación, los de corte constructivista ofrecen varios caminos alternativos de conducción del proceso, es decir, diversas rutas y patrones a seleccionar. El desarrollo de nuevos modelos de diseño de aprendizaje exige un preciso control de calidad, lo que implica un riguroso trabajo de evaluación y colaboración. En este artículo se asumen estos enfoques, donde el profesor se contempla como un mediador y su metodología debe promover el cuestionamiento de los fenómenos y la investigación. Así el protagonismo es del que aprende, y el docente deviene facilitador del aprendizaje.

\subsection{3- Tendencias y herramientas actuales del diseño de aprendizaje}

El aprovechamiento adecuado de las TIC en el ámbito educativo es crucial para facilitar a los educadores las herramientas necesarias para impactar creativamente el proceso de enseñanza-aprendizaje, permitiéndoles superar los retos y desafíos que les demanda un entorno disruptivo y global para avanzar con éxito hacia una sociedad basada en conocimiento (UNESCO, 2009). Se pretende por tanto aprovechar este enorme potencial para transformar la educación con ayuda de estas tecnologías, en consonancia con los cambios acelerados que estas producen en la sociedad y teniendo en cuenta que

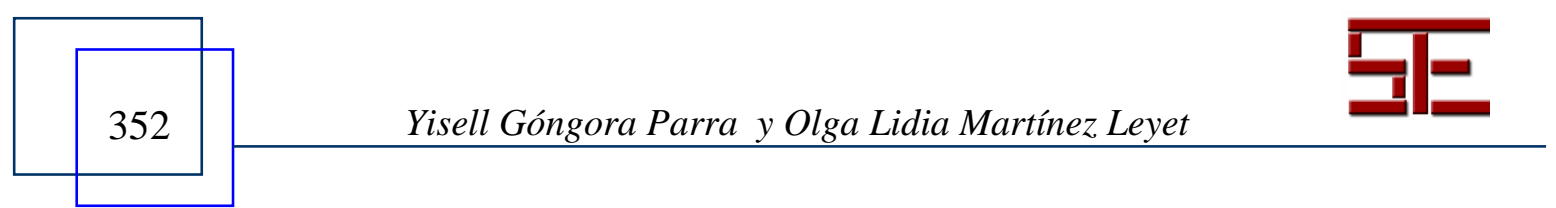




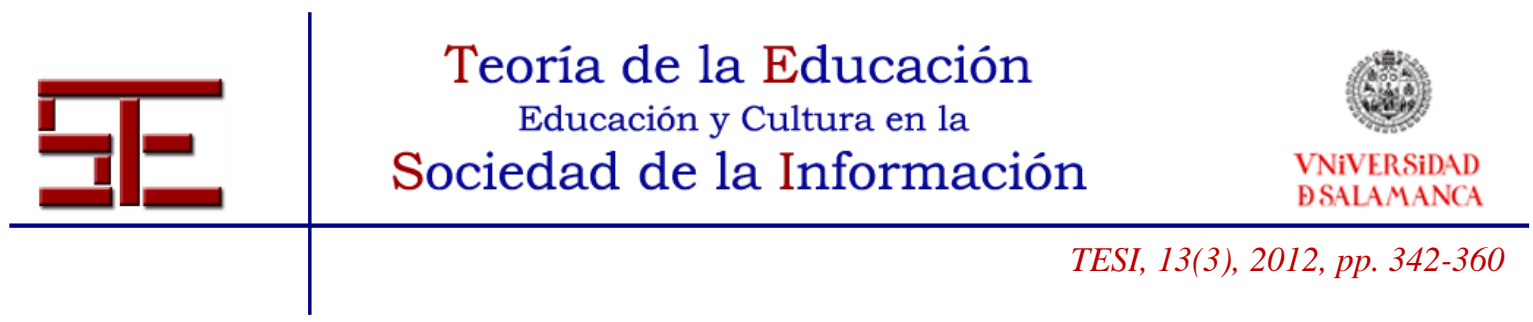

la enseñanza actual ha traspasado las paredes del aula física y ocurre en prácticamente cualquier ambiente de aprendizaje. La Comisión Europea presenta los principios, objetivos y líneas de acción del e-learning, definidos como "la utilización de las nuevas tecnologías multimediales y de Internet, para mejorar la calidad del aprendizaje facilitando el acceso a recursos y servicios, así como los intercambios y la colaboración a distancia" (Zapata, 2005).

Los materiales y programas que se diseñen deben promover el desarrollo del pensamiento lógico y habilidades más efectivas de gestión de la información y el conocimiento; acordes con los enfoques actuales de la cultura participativa, el conocimiento, contenido, medios y aprendizaje abiertos (Goodyear, 2008; Conole, 2009), las tecnologías libres (Koper, 2008; Bennet, 2009), el e-learning (Holmberg 1977, Keegan, 1990, Sun et al., 2005) y los entornos virtuales de aprendizaje (Harasim et.al., 2000; Salmon 2002; García, 2007).

El diseño de aprendizaje basado en tecnologías (Computer Based Learning Design) optimiza el trabajo del equipo de docentes que laboran en el diseño del curso o material educativo. En la actualidad, el gran número de herramientas tecnológicas para el proceso de diseño de aprendizaje genera un cambio especialmente relevante para los profesores, quienes asumen la tarea de crear y diseñar, pero también de seleccionar y evaluar un gran número de materiales para ser utilizados por los estudiantes en entornos virtuales.

Los nuevos modelos apuntan a una visión más abierta y libre del aprendizaje y a un mayor protagonismo por parte del estudiante. Paralelo a la proliferación de estos, han surgido nuevas herramientas tecnológicas que crean nuevos y más flexibles espacios para el diseño, creación y gestión de recursos y objetos de aprendizaje. Entre estas se pueden mencionar Compendium LD, Reload, ReCourse, EXelearning, Cloudworks, Learning Activities Management Systems (LAMS), MOT+, COLLAGE, OPENGLM, Coursew Are Development Methodology for Open instructional Systems (CADMOS) Instructional Management Systems - Learning Design (IMS-LD), entre otros. Se hace necesario por tanto formar profesionales hábiles en su uso para el diseño de alternativas de aprendizaje para los nuevos y variados contextos.

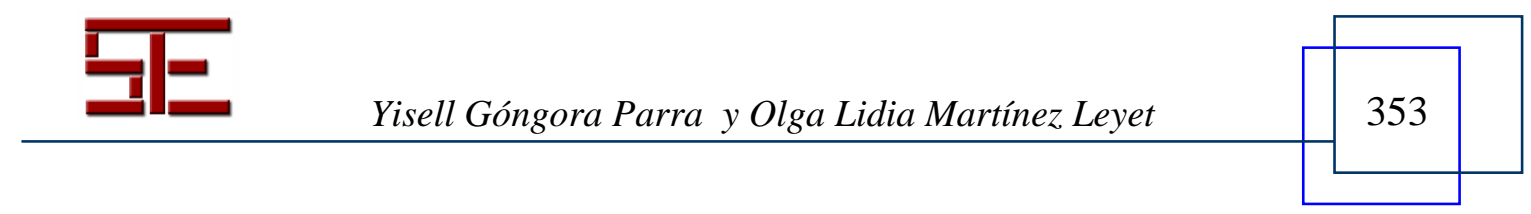




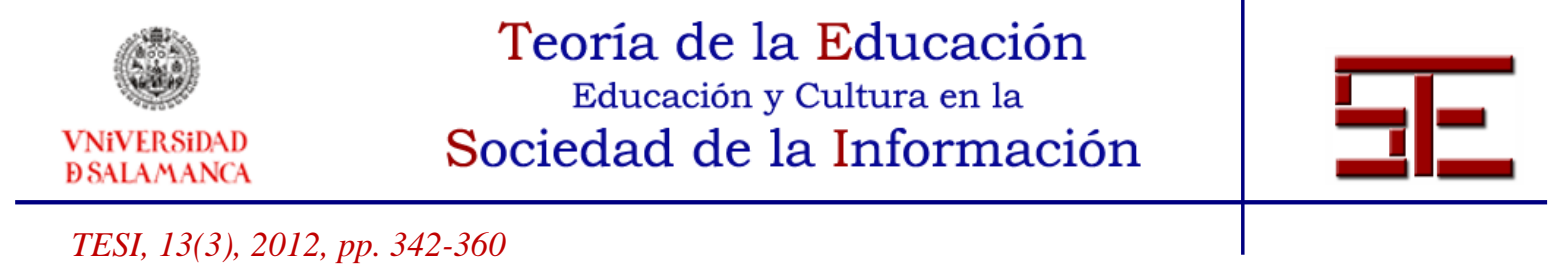

Entre las ventajas de estas tecnologías de nueva generación podemos mencionar que la mayoría es de código abierto y se puede usar para la creación de mapas conceptuales, actividades de aprendizaje y para la planificación y diseño de cursos. Además, generalmente se puede incluir el código HTML que se genere en la plataforma Moodle o en cualquier otra plataforma de e-learning. Estas herramientas permiten el diseño de rutas de aprendizaje personalizadas para cada estudiante una vez que los conceptos del proceso han sido definidos. Las rutas de aprendizaje pueden incluir aquellos recursos o actividades que permitan a los estudiantes llevar a cabo un autoaprendizaje adaptivo. (Moreno, 2007).

Sin embargo, algunas de sus limitaciones consisten en que son aplicaciones de escritorio, no compatibles con los estándares IMS-LD, lo que limita la reusabilidad de sus patrones, tal es el caso del Compendium LD (Katsamani \& Retalis 2008). La herramienta Reusable eLearning Object Authoring and Delivery (Reload) permite el diseño y creación de contenidos educativos, sobre todo para su empaquetamiento en ficheros comprimidos. El uso de esta herramienta y su sucesora ReCourse para el diseño de un aprendizaje constituye un potencial expresado en su usabilidad e interoperabilidad. Sin embargo, son también herramientas de escritorio y esto limita su accesibilidad. Más específicamente, Reload permite la creación y edición de paquetes y la inserción de metadatos conforme a las especificaciones de ADL e IMS. Utiliza el estándar SCORM y permite la comunicación con los LMS (Griffiths D et al., 2010).

EXeLearning es un redactor de XHTML para generar recursos y contenidos para el Aprendizaje Electrónico (e-learning), un ambiente que asiste a profesores y académicos en trazar y publicar cursos en Internet sin la necesidad de aprender HTML o XML, desarrollado por la Auckland University of Technology y la Tairawhiti Polytechnic. Con esta herramienta los usuarios pueden desarrollar estructuras de capacitación que satisfagan sus necesidades de entrega del contenido y crear recursos más flexibles y fáciles de mantener; sin embargo, no es un Learning Management System (LMS) sino un ambiente para trazar el contenido de aprendizaje basado en web.

Otra herramienta a considerar debido a su uso e impacto es Cloudworks, desarrollada por un equipo de la Open University. Se crea con el objetivo de que los equipos de profesores de la OU que diseñan cursos y actividades de aprendizaje compartan sus prácticas e ideas y colaboren en el diseño de recursos y materiales para la enseñanza

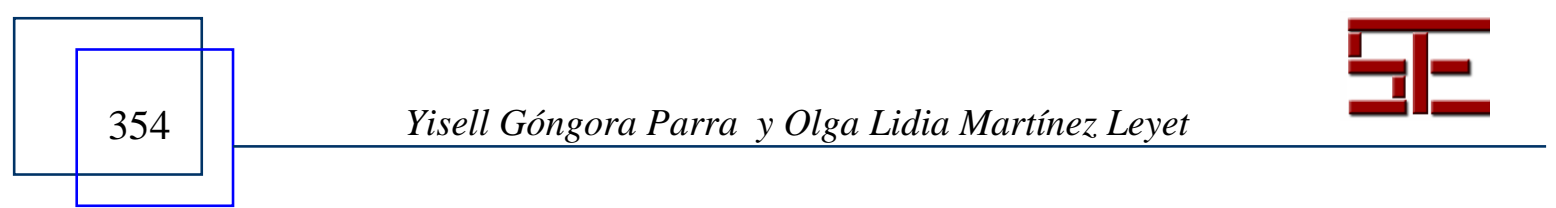




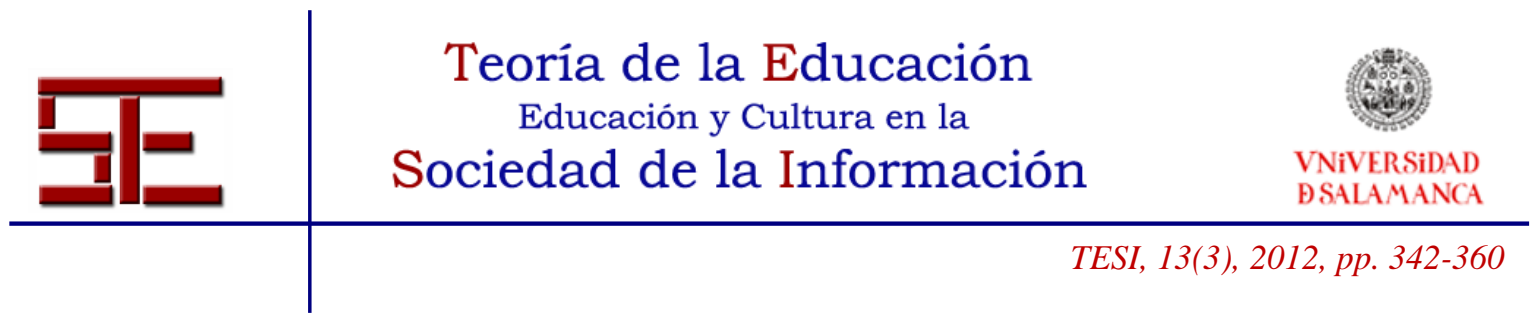

(Conole \& Culver 2008). Los creadores de la herramienta emplearon principios de la Web 2.0 y dedican especial atención a la retroalimentación de las experiencias de los usuarios finales. Permite además que el diseño se realice en varios formatos como CompendiumLD maps y LAMS.

El Instructional Management System - Learning Design (IMS-LD) surge a raíz de las limitaciones pedagógicas del IMS, con el objetivo de crear una especificación para la definición de aspectos pedagógicos, pero ya que el Educational Modelling Language (EML) existía y funcionaba decidieron adaptarlo en lugar de crear una especificación totalmente nueva. Los aspectos fundamentales del IMS-LD son los siguientes:

- ofrece soporte para múltiples alumnos, y contempla la comunicación entre ellos

- representa el papel de profesor

- permite combinar recursos educativos con actividades pedagógicas, y con las interacciones entre personas en diferentes roles (Griffiths et al., 2010).

Esta precisamente es una de las especificaciones usadas en la actualidad en la Universidad de las Ciencias Informáticas, donde se desarrollan herramientas de autor para la producción de cursos y materiales de aprendizaje y de esta forma contribuir a las necesidades educacionales de la enseñanza en sus distintos niveles.

La formación de profesionales preparados, capaces de usar las herramientas para crear materiales educativos con eficiencia, calidad y didácticamente coherentes requiere la correcta capacitación del diseñador de aprendizaje, rol definido dentro de los equipos de desarrollo de software educativo. La UNESCO (2008) define, entre sus estándares de competencias en TIC para docentes, cinco competencias básicas y la primera de ellas es que el profesional de hoy debe ser "competente para utilizar tecnologías de la información".

Siguiendo esta línea, la universidad pretende formar a un profesional capaz de realizar diseños efectivos de aprendizaje, seleccionando los modelos más eficaces, utilizando las herramientas tecnológicas adecuadas y aplicando las teorías de aprendizaje a los más

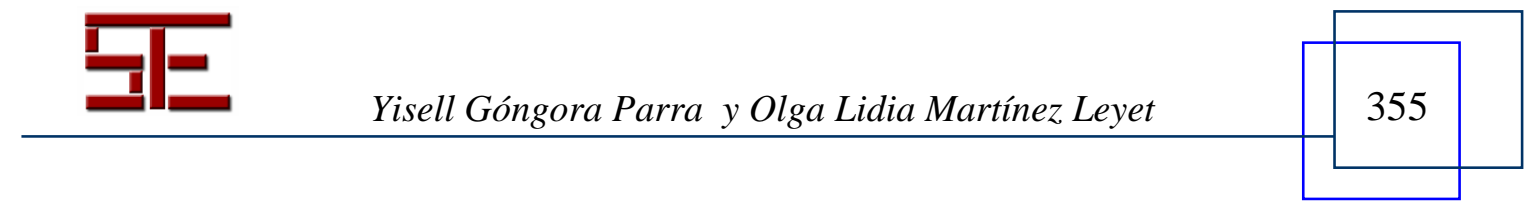




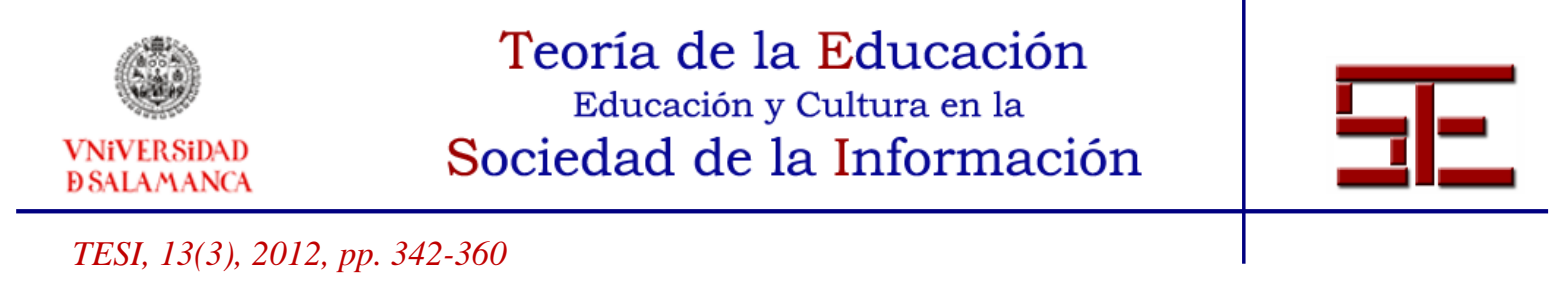

diversos contextos educativos. Sin embargo, hay carencias aún referentes a que el proceso de capacitación de diseñadores de aprendizaje se ofrece más como preparación en el trabajo con software específico y no como metodologías generales para su adaptación a diseños particulares. Existe además una marcada tendencia a ver la creación de materiales educativos como un proceso de dos vertientes: la pedagógica, separada de la informática y no con la imbricación necesaria, unificada en el rol del diseñador de aprendizaje.

Para atender a estas necesidades, las herramientas de autor creadas en la universidad que incorporan el módulo de IMS-LD se utilizan para el diseño de cursos y actividades de aprendizaje, con una interfaz gráfica amigable y pueden ser usadas por docentes de las más diversas materias. El principal objetivo de estas herramientas es facilitar la creación de objetos de aprendizaje (OA) empleando el estándar SCORM en su versión 1.2, garantizando así la interoperabilidad y reusabilidad de los recursos educativos en diferentes aplicaciones que utilicen este estándar. Otra de las ventajas es la posibilidad de crear plantillas, que luego pueden publicarse para su uso por parte de otros diseñadores de aprendizaje. A los OA que son creados, se les incorporan metadatos que describen algunos de sus elementos, facilitando así la búsqueda, identificación y reusabilidad en repositorios de objetos de aprendizaje.

Este editor permite a los autores de contenidos compartir sus diseños, por lo que tiene un valor significativo según los nuevos estándares educacionales. Las nuevas tendencias del diseño de aprendizaje abogan por entornos de creación autónomos, colaborativos, participativos, situados, abiertos y dialógicos.

\section{CONCLUSIONES}

- Los modelos de diseño de aprendizaje basados en teorías constructivistas son más adecuados para los nuevos contextos educativos y ofrecen más oportunidades para diseñar acciones formativas que permitan el alcance de competencias profesionales.

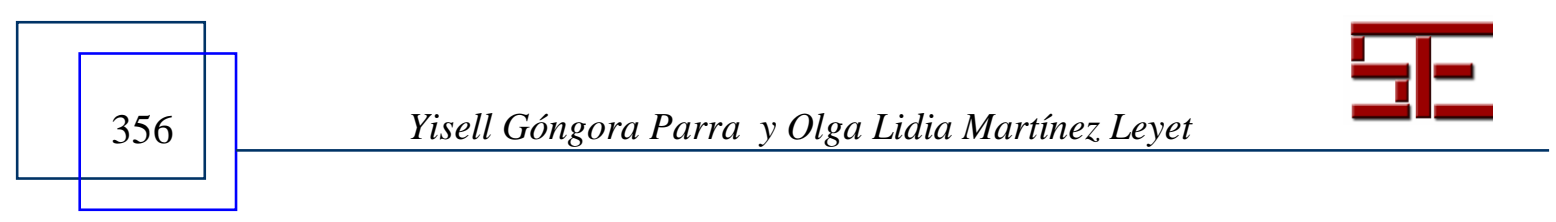




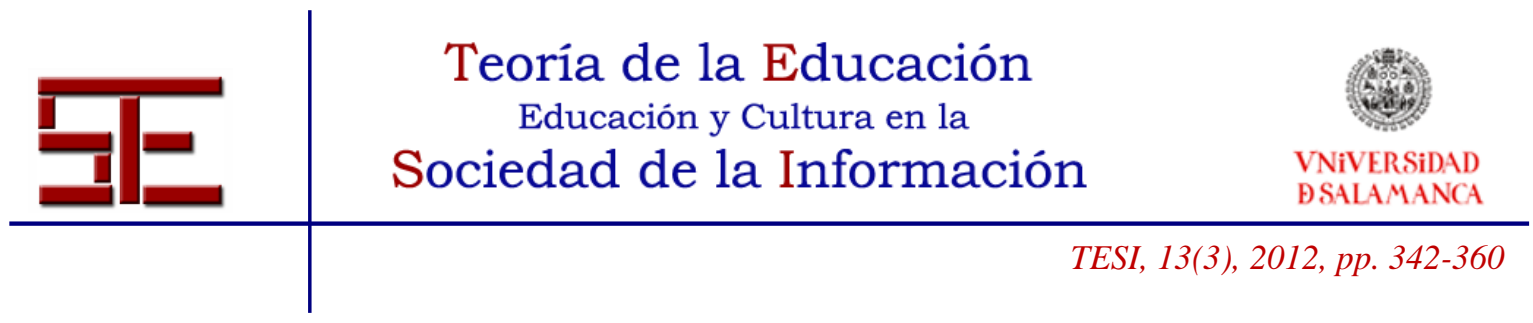

- Al utilizar los modelos que señalan la enseñanza basada en problemas el estudiante estará mejor preparado para aplicar sus conocimientos a situaciones nuevas y cambiantes.

- Los modelos de diseño de aprendizaje pueden ser una guía valiosa para el proceso, pero hay que tener en cuenta las características de la audiencia y de cada entorno de aprendizaje, que lo hacen único.

- El desarrollo actual del diseño de aprendizaje supone una ruptura de posturas rígidas y una búsqueda de nuevas alternativas para optimizar el proceso frente a los cambios y las necesidades de la educación.

- Los estándares y herramientas de diseño de aprendizaje más usados en la actualidad son aquellos que permiten la interoperabilidad y reusabilidad de los recursos, así como el compartir los diseños y plantillas.

\section{BIBLIOGRAFÍA}

Ballstaedt (2000). Puesta en práctica de entornos de aprendizaje: la fase de implementación. Extraído el 12 de enero, 2012, de http://cinterfor.org.uy/public/spanish/region/ampro/cinterfor/publ/inwent/pdf/cap_5.pdf

Conole, G. (2009). The Role of Mediating Artefacts in Learning Design. UK: The Open University.

Dick, W. y Carry, L. (2004). Systematic Design of Instruction. State University.

Fernández, N. (2004). Factores psicopedagógicos de influencia en la eficiencia terminal de actos académicos de educación continua por internet. Extraído el 14 de diciembre, 2010, de http://www.e-continua.com/documentos/disenio_instruccional.pdf.

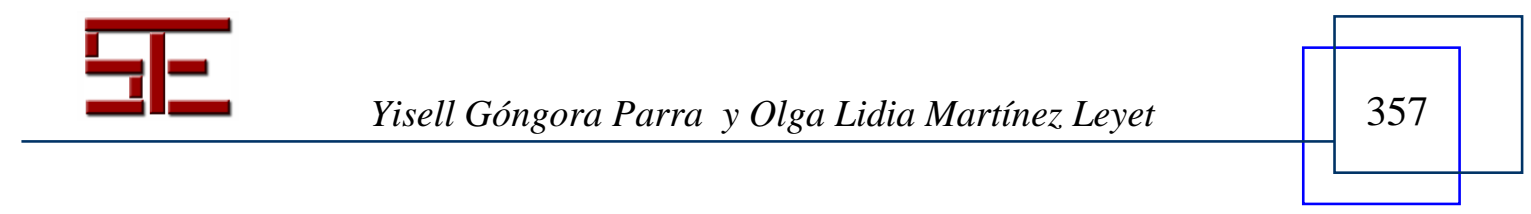




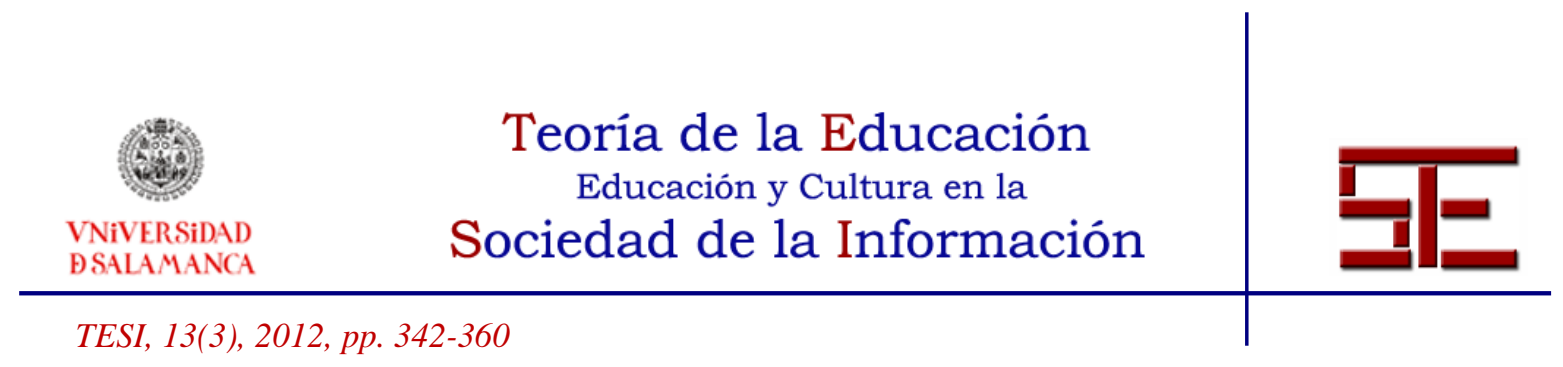

Gerlach, V. S. \& Ely, D. (1980). Teaching \& Media: A Systematic Approach. Second edition. Englewood Cliffs, New Jersey, Prentice Hall Inc.

González, O. (2007). Materiales didácticos para el desarrollo de competencias de lectura técnica en inglés. Universidad Juárez Autónoma de Tabasco.

Goodyear, P. (2009). Patterns and Pattern Languages in Educational Design. Australia: University of Sydney.

Griffiths, D. (2010) La aportación de IMS Learning Design a la creación de recursos pedagógicos reutilizables. Universitat Pompeu Fabra.

Guàrdia, L. (2002). Diseño instruccional y objetos de aprendizaje; hacia un modelo para el diseño de actividades de evaluación del aprendizaje on-line. Extraído el 26 de enero, 2012, de http://www.online-educa.com/en/.

Harasim, L. (2000). Educational applications of computer conferencing. Journal of Distance Education.

Heinich, N. (1999). Instructional Media and Technologies for Learning. Extraído el 12 de enero, 2010, de http://disenoinstrucional.files.wordpress.com/2007/09/theassuremodel.

Jonassen, D. (1999). Designing constructivist learning environments. Instructional Design Theories and Models. vol. 2. Lawrence Erlbaum.

Kemp, J. (1985). The Instructional Design Process. Estados Unidos: Harper Collins

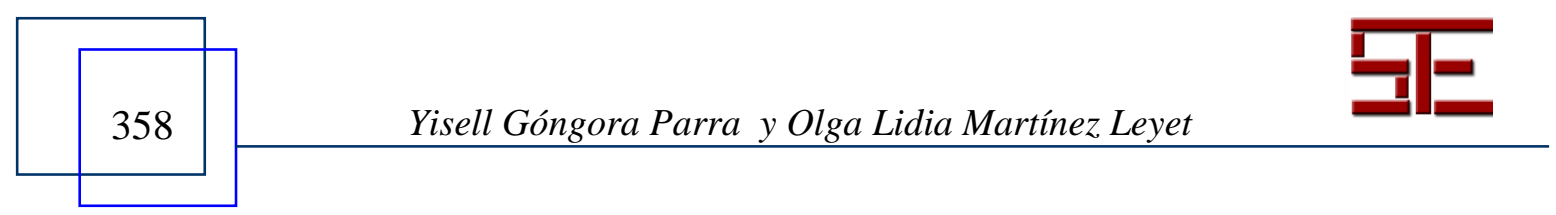




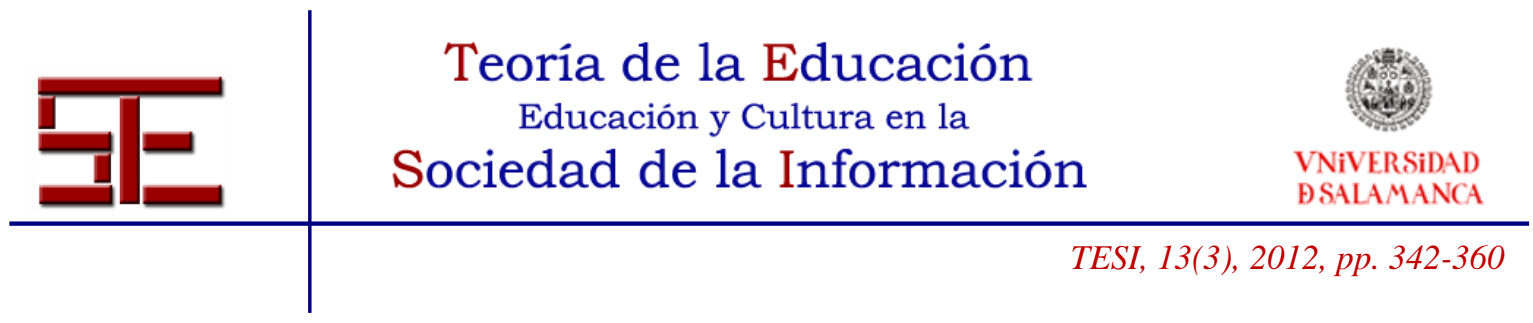

Koper, R. y Tattersall, C. (2005). Learning Design: A Handbook on Modelling and Delivering Networked Education XXVIII. ISBN: 978-3-540-22814-1

Lockyer, L. y Bennet, S. (2009). Handbook of Research on Learning Design and Learning Objects: Issues, Applications, and Technologies. Australia: University of Wollongong Information Science.

Mayer, R. (2006). Learning Strategies for making sense out of expository text: The SOI model for guiding the cognitive processes in knowledge construction. Estados Unidos: Departamento de Sicología. Universidad de California.

McGriff, S. (2007). Instructional Systems, ADDIE MODEL. College of Education, Penn State University.

Merrill, M. D. (2000). Component Display Theory. Instructional Design Theories and Models. Hillsdale NJ: Erlbaum Associates.

Moreno, L. (2007). Hacia un sistema inteligente basado en Mapas Conceptuales evolucionados para la automatización de un aprendizaje significativo. Extraído el 12 de enero, 2010, de http://bioinfo.uib.es/ joemiro/aenui/procJenui/Jen2007/mohaci.pdf.

Ruiz, J. M. (2007). Diversidad en la organización del contenido matemático en planes de estudio universitarios. Revista Iberoamericana de Educación (ISSN: 1681-5653) n. ${ }^{\circ}$ 42/4 - 10 de abril de 2007. Cuba: Organización de Estados Iberoamericanos para la Educación, la Ciencia y la Cultura (OEI)

Ryder, M. (2006). Modelos educacionales del diseño. Universidad de Colorado en Denver.

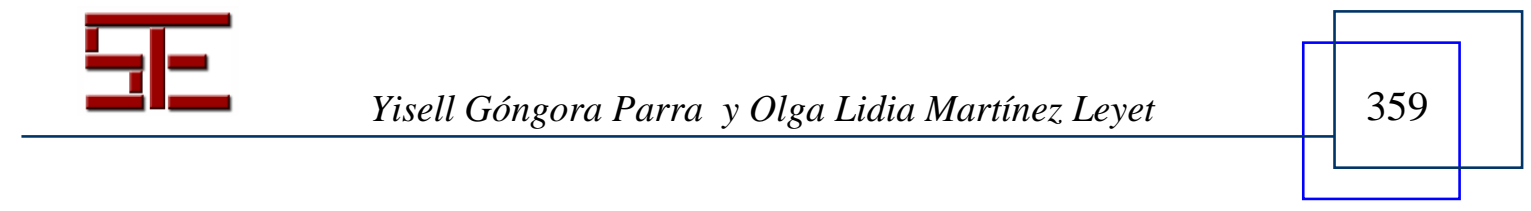




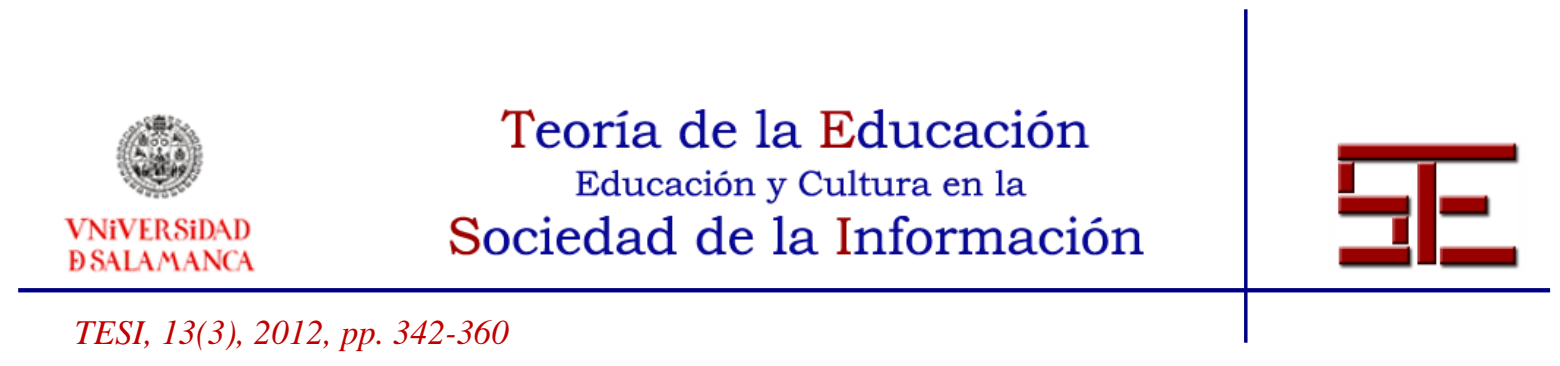

Seels, B. (2008). Historia del Diseño Instruccional. Extraído el 12 de enero, 2010, de http://medusa.unimet.edu.ve/programacion/dpto/PagDI/Principal/Historia.html.

Tyler, R. (2009). Teoría y Práctica del Diseño Instruccional. Nacimiento del objetivo de aprendizaje. Extraído el 13 de diciembre, 2011, de http://instruccioneseducativas.hernanramirez.info/?p=19.

Vázquez, L. (2010). Diseño Instruccional. Extraído el 12 de diciembre, 2011, de www.mse.buap.mx/recursos/diseno_instruccional/diseo_instruccional.

Velasco, A. (2007). Algo sobre la robótica pedagógica. Extraído el 12 de diciembre, 2011, de http://ana-educadistancia.blogspot.com/2007/03/algo-sobre-la-robticapedagogica .

Walsh, D. (2006). Cognitive Learning Theory. Extraído el 15 de diciembre, 2011, de http://www.slideshare.net/danielle_walsh/cognitive-learning-theory-presentation.

Zapata, M. (2005). Educación a distancia: El aprendizaje, ¿objeto u objetivo? Extraído el 2 de febrero, 2012, de www.um.es/ead/mex2005/presentacionMonterrey.ppt.

Para citar el presente artículo puede utilizar la siguiente referencia:

Góngora Parra, Y. y Martínez Leyet, O. L. (2012). Del diseño instruccional al diseño de aprendizaje con aplicación de las tecnologías. Revista Teoría de la Educación: Educación y Cultura en la Sociedad de la Información. 13(3), 342-360 [Fecha de consulta: $\mathrm{dd} / \mathrm{mm} / \mathrm{aaaa}]$.

http://campus.usal.es/ revistas_trabajo/index.php/revistatesi/article/view/9144/9377

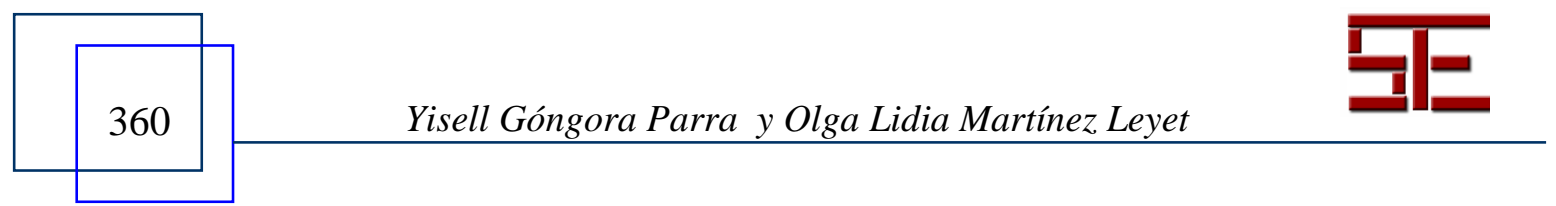

